\title{
Maximizing the Revenues of Data Centers in Regulation Market by Coordinating with Electric Vehicles 类
}

\author{
Marco Brocanelli, Sen Li, Xiaorui Wang, Wei Zhang \\ Department of Electrical and Computer Engineering, The Ohio State University, USA
}

\begin{abstract}
Frequency regulation is a major market service to reduce the undesired imbalance between power supply and demand in the power market. In order to participate in the regulation market, both the supply and demand sides need to be capable of flexibly adjusting their power generation and consumption, respectively. As the scale of Internet data centers is increasing rapidly, their significant power consumption has enabled them to become an important player in the regulation market for maximized profits and thus minimized operating expenses. On the other side, Plug-in Hybrid Electric Vehicles (PHEVs) have also recently been identified as a major participant in the regulation market, due to their large power demand for battery charging.

In this paper, we propose a novel power management scheme that jointly leverages a data center and its employees' PHEVs to 1) maximize the revenues that the data center receives from the regulation market, and 2) get the PHEVs charged at no expense to their owners. Our scheme features a two-level hierarchical power control design. At the first level, our scheme interacts with the regulation market to provide information about the data center power consumption on an hourly basis. At the second level, the scheme decides the power budgets for the servers and UPS in the data center, as well as PHEVs, in real time, to follow the given regulation signal. In addition, we show how to leverage the Thermal Energy Storage (TES) tanks available in many data centers to adapt the cooling power consumption for better management of the data center power demand and further increased regulation revenues. We evaluate the proposed scheme with real-world workload and regulation traces. The results show that our scheme performs a high-quality regulation service. As a result, the proposed scheme outperforms several commonly used baselines by having higher regulation revenues, and so lower operating expenses, for the data center. Finally, we analyze the cost savings of the PHEV owners, throughout the lifetime of the PHEVs, by getting their batteries charged at no expense.
\end{abstract}

Keywords: Frequency Regulation, Electric Car, Data Center.

\section{Introduction}

Frequency regulation is a major market service [1] to reduce the undesired imbalance between power supply and demand in the power market. In order to participate in the regulation market, both the supply and demand sides need to be capable of flexibly adjusting their power generation and consumption, respectively. Every few seconds the regulation market sends a regulation signal to the regulation assets according to an agreed regulation capacity [2], [3]. Based on capacity range and tracking performance of the signal, participants receive compensation from the market [4], [5], [6]. Internet data centers [7], [8] and Plug-in Hybrid Electric Vehicles (PHEVs) [9], [10], [11] have been recently identified as eligible participants in the regulation market. However, existing work does not consider the possibility to join data centers and PHEVs to maximize frequency regulation profits.

\footnotetext{
This work was supported, in part, by NSF CAREER Award CNS-1143607.

Email addresses: brocanelli.1@osu .edu (Marco Brocanelli), 1i.2886@osu.edu (Sen Li), xwang@ece. osu.edu (Xiaorui Wang), zhangeece.osu.edu (Wei Zhang)
}

Modern data centers can participate in the regulation market thanks to their ability to flexibly change power consumption. For example, they can 1) change server power consumption with Dynamic Voltage Frequency Scaling (DVFS) [12], 2) manage charge and discharge cycles of Uninterruptible Power Supply (UPS) batteries [13], and 3) turn on/off servers via dynamic capacity provisioning [14]. Moreover, data centers account for $1.5 \%$ of total energy demand in US [15]. The percentage is expected to grow up to $8 \%$ by 2020 [16]. As the scale of Internet data centers is increasing rapidly, their significant power consumption has enabled them to become an important player in the regulation market for maximized profits and thus minimized Operating Expenses (OpEx).

Another way to mitigate the imbalance in the power grid, is offered by electric vehicles. The introduction of these cars is mainly driven by the price increase and by the limited quantity of fossil fuel. PHEVs can play an important role to maintain frequency stability, due to their large power demand for battery charging [10], [9]. These cars are typically equipped with a $2 \mathrm{kWh}$ to $10 \mathrm{kWh}$ battery capacity. For example, if 50 vehicles are charging at the same time and in the same place, there are up to $500 \mathrm{kWh}$ to use for regulation. Assume that in a remote 
future everyone owns an electric car. A data center's parking lot may have potentially 50 to 200 small batteries to exploit for frequency regulation [17], [18]. The contribution of electric cars, is useful for data centers to further widen the regulation capacity and track the regulation signal. In addition, employees have the opportunity to receive a free charge of their vehicles.

In this paper, we propose a novel two level hierarchical power management scheme that jointly leverages a data center and its employees' PHEVs to 1) maximize the revenues that the data center receives from the regulation market, and 2) get the PHEVs charged at no expense to their owners. At the first level, our scheme interacts with the regulation market to provide information about regulation capacity and baseload on an hourly basis. At the second level, the scheme decides the power budgets for servers, UPS, and PHEVs, in real time, to follow the given regulation signal. Our algorithm 1) ensures 24 hours of regulation for maximized revenues, 2) respects response time of workload and battery lifetime constraints, 3) ensures an acceptable final state of charge for the vehicles, and 4) offers high quality regulation service. In addition, because the cooling system today represents a big portion of data center power demand, we study how Thermal Energy Storage (TES) devices equipped in many data centers, such as ice tanks or water tanks, can be integrated in the power management scheme to further increase regulation revenues.

The rest of the paper is organized as follow: Section 2 discusses the related work. Section 3 gives some background about regulation market and PHEVs. Section 4 describes our control architecture. Section 5 gives insights about its different components. Section 6 describes how to exploit thermal energy storage in the regulation problem. Section 7 describes the trace files and the baselines used in our simulations. Section 8 shows the results and Section 9 concludes the paper.

\section{Related Work}

Numerous approaches for data center power management have been explored in literature. Lefurgy et al. [19], Wang and Chen [20], and Wang et al. [12] explore the potential of DVFS to accurately track a desired power consumption at server, cluster, and data center level, respectively. Ahmad and Vijaykumar [21] choose which servers to turn on in order to optimize the temperature within a data center. Liu et al. [14] adjust the number of active servers to minimize OpEx. Kontorinis et al. [13] study UPS batteries in different configurations to track a desired power budget at data center level. Urgaonkar et al. [22] charge and discharge batteries according to fluctuation of energy price to minimize OpEx. Different from these papers, we propose to exploit knobs of data centers jointly with PHEVs, to participate in the regulation market.

Regulation market is a service to maintain the instantaneous balance between demand and generation to ensure power grid reliability. Traditionally this service is mainly provided by coal fired generators that come with heavy cost associated with degraded heat rate. Moreover, generators lose opportunities in producing electricity. Some non-traditional resource is also proposed in literature as for example commercial buildings [23]

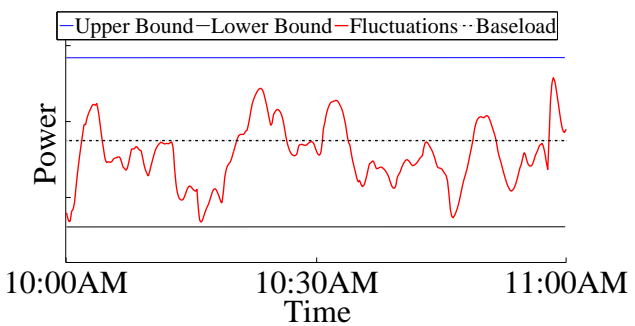

Figure 1: The regulation signal leads to fluctuations of power consumption around the power baseload.

and aggregated residential loads [24]. However, the long response time of commercial buildings limits the potential of these structures as a regulation resource. In addition, the control of residential loads requires expensive metering and communication infrastructures; the expenses of these advanced instruments leave the regulation effort unrewarded. Our paper instead, explores the potentialities to integrate modern data center and electric cars for regulation service.

Few other studies attempt to involve data centers in the regulation market. Wang et al. [7] explore the integration of multiple data centers in the demand response market by migrating virtual machines. Aikema et al. [8] study how a data center can provide different services of the ancillary market with DVFS to reduce costs. Different from existing work, our solution exploits multiple knobs (e.g., DVFS, UPS, and capacity provisioning) of the data center jointly with PHEVs charging rate, to provide high quality regulation service.

Other research projects use PHEVs to minimize frequency imbalance. Tuffner and Kintner-Meyer [10] and Li et al. [25] schedule a smart charge of PHEV batteries to minimize the fluctuations of wind energy. Kempton et al. [9] equip PHEVs with a special mechanism called V2G. This mechanism, besides simply charging batteries, allows the grid to discharge them. However, wind energy is not the only cause of frequency fluctuations and not all the electric cars are equipped with the V2G. Different from previous work, our algorithm directly interacts with regulation market to offer regulation service. Additionally, we only charge the PHEV cars once per day to preserve battery lifetime and to refer to a more common case.

\section{Background}

\subsection{Regulation Market}

Regulation Market is a kind of ancillary service [26]. It has been created to stabilize the grid frequency (around $60 \mathrm{~Hz}$ ) when an imbalance between generation and demand occurs. At the beginning of each operation hour, the regulation assets send the baseload power and regulation capacity to the market to characterize the agreed regulation capacity range. Figure 1 illustrates power baseload and bounds. Regulation capacity is the absolute difference between baseload and higher (or lower) bound. Within each operation hour, participants in regulation service, receive from the market the regulation signal with time step between 2 seconds to 60 seconds. This signal is normalized between -1 and 1 and is then elaborated by the assets. For example, if an asset commits $0.1 \mathrm{MW}$ capacity with baseload 
$1 \mathrm{MW}$, then a regulation signal with value 0.8 means that the asset has to consume or generate $1.08 \mathrm{MW}$ of power.

Data centers are eligible assets for regulation service because of their high power flexibility and their growing power consumption. Our work shows how to decrease data center's OpEx with frequency regulation without any loss in reliability and performance. Moreover, we jointly manage PHEV cars with data centers to further maximize regulation profits.

\subsection{PHEV Cars}

PHEVs [27] differ from conventional cars because they add an electric engine, which does not use fuel, to the normal one. These vehicles indeed, are equipped with a battery pack that feeds the electric engine. The combination of the two engines drastically decreases fuel consumption and allows to maintain a similar driving range of normal cars. If the battery is depleted indeed, one can still drive with the normal fuel. Typically the lifetime of a PHEV depends on how the battery capacity is dissipated as time goes by. The capacity feeding rate varies, depending on ambient environment, driving patterns and charging scenario. On average however, batteries of PHEVs last about 15 years [28], [29]. Clusters of electric vehicles can act in charging stations as an energy conversion system to mitigate power grid imbalance [25], [27], [11], by altering the charging rate or deferring the charging time.

Literature provides modeling approaches with statistics that capture driving schedule and temporal distribution of PHEVs [30]. However in this paper we assume randomly distributed arrival and departure times between 7-9 AM and 3-5 PM, respectively. These intervals typically correspond to the working hours of the data center. This assumption can be easily relaxed to a more general case, which is part of our future plan. We assume that the number of employees in a data center is from 50 to 200 ([17], [18]), though some big IT companies often have large office campuses collated with their data centers. Other types of electric cars today in the market, are the Battery Electric Vehicles (BEV, no fuel engine) and Hybrid Electric Vehicles (HEV, no plug-in). Contrary to HEVs, BEVs can be used as well in this paper.

\section{System Architecture}

Figure 2 shows the system architecture. In this section we introduce the power management system to describe its twolevel hierarchy. Furthermore, we briefly highlight how our system coordinates regulation market, data center and PHEVs, to enable data centers to provide regulation service. Because sales of electric cars are expected to grow in the future [31], we assume a widespread use of PHEVs. We also assume that data centers connect charging stations to their power facilities. In this scenario, employees are allowed to plug their electric vehicles directly to the data center during working hours.

\subsection{Power Management System Hierarchy}

The main component of the scheme is a two layer hierarchical power management system. At the first level, the system

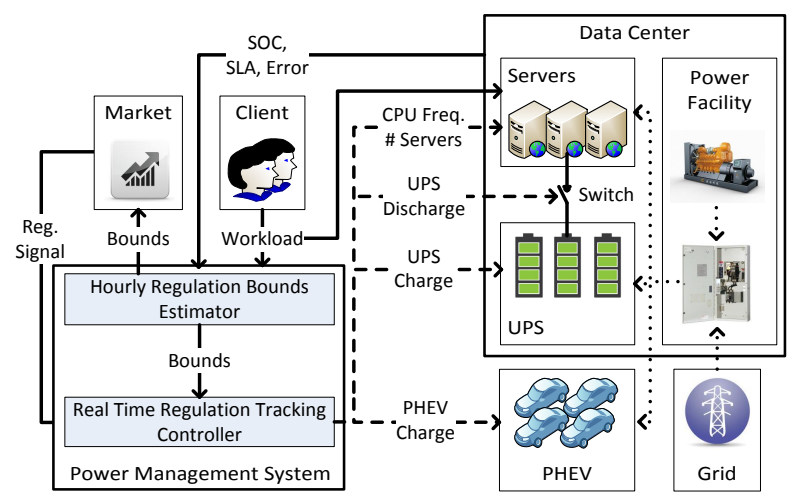

Figure 2: The power management system interacts with regulation market, data center and PHEVs, to enable data centers to provide regulation service.

unit has a hourly regulation bounds calculator. At the second level, there is a real time regulation tracking controller. These two subsystems work with different time periods and variables. The bounds calculator is invoked once per hour. This component establishes the number of active servers and calculates the regulation capacity to communicate to the regulation market (see Section 3.1). The tracking controller has an invocation time of 4 seconds. This component calculates CPU frequencies of servers as well as charging/discharging rates of batteries to track the regulation signal. The invocation periods are justified by the market rules as described in Section 3.1.

\subsection{Hourly Regulation Bounds Calculator}

The challenges for the calculation of the number of active servers and size the regulation bounds are two-fold. First, the algorithm has to comply with a set of constraints that influence control inputs. Second, the algorithm needs to interact with the unpredictable nature of the regulation signal and fluctuations of workload. The target is to maximize regulation revenues. In this section we give a high level description of how the bounds calculator decides number of servers and bounds, to comply with constraints and fluctuating signals.

The first important aspect to consider are the constraints of servers and batteries. The active servers are allowed to change power consumption as long as Service Level Agreements (SLA) are satisfied. Batteries experience a lifetime reduction in case of frequent charging/discharging cycles or in case of excessive depletion [32]. Moreover, reliability needs to be preserved because in case of outage of the power utility, UPS batteries need to sustain the entire data center.

The bounds calculator makes decisions on the number of active servers $N_{a}$ based on the estimated requests average value $\lambda_{\text {mean }}$. Predicting average workload indeed, is simpler than predicting instantaneous workload. In order to make the controller robust on workload variations around $\lambda_{\text {mean }}$, we calculate the number of active servers proportionally to a scaling factor $\gamma$. This solution allows to respect SLAs requirements. The choice of $N_{a}$ is neither too conservative nor too aggressive, because based on average workload. 
Batteries' lifetime is another constraint. To avoid UPS batteries experience frequent charging/discharging cycles, we ensure a maximum of 1 discharge and 2 charges during the day. The bounds calculator divides UPS into two groups. The charge set $R$ and the discharge set $D$. Depending on initial energy level, UPS batteries are placed, at the beginning of the day, in the set $R$ or $D$. Every UPS in the set $R$, if never discharged, is allowed to switch in $D$ when fully charged; otherwise, the UPS becomes unavailable until the next day. Similar case for switching from $D$ to $R$, with the exception that the maximum Depth Of Discharge (DOD) has to be respected. Moreover, the bounds calculator estimates the time periods of the day in which batteries should finish every cycle. This rough estimation, allows to distribute UPS energy through the whole day, such that revenues are maximized. While UPS can be part of both the sets $R$ and $D$, PHEV batteries cannot be discharged. The technology V2G [9], which allows the grid to discharge electric cars, is not widespread and is expensive. Therefore, PHEVs are placed in $R$ and become unavailable once full. Their availability depends also on the working hours.

The second problem that the bounds calculator needs to address, is the unpredictability of the regulation signal $P_{\text {reg }}$. Two issues stem from this unpredictability. First, how to ensure high tracking quality of $P_{r e g}$. Second, how to ensure a good final energy level of PHEVs and exploit all the energy in the batteries. The bounds calculator needs to submit the regulation capacity and the baseload before each operation hour. The tracking of the regulation signal is done by managing server's frequencies and batteries' feeding rates. Hence, in order to ensure high regulation quality, the bounds calculator should know exactly the amount of energy necessary to track the regulation signal $P_{\text {reg }}$. Although this knowledge is impossible, the regulation signal 1) complies with regulation bounds, and 2) has a statistical average value equivalent to the baseload. Given these information, the bounds calculator conservatively calculates the regulation capacity based on a worst case regulation signal $P_{\text {reg }}^{\text {Wort }}$. Therefore, given $P_{r e g}^{W o r s t}$ and $N_{a}$, the bounds calculator determines the maximum allowed power variation bounds of servers. Batteries' energy is useful to widen the server bounds (e.g., the regulation capacity). However, the bounds calculator needs to ensure high final SOC of PHEVs. In addition, it has to ensure a correct redistribution of UPS energy for the whole day to maximize regulation revenues. To solve these two issues, the contribution of batteries to the regulation bounds is calculated proportionally to the State of Charge (SOC) of batteries, and inverse proportionally to the remaining charging/discharging time period of each battery. The power baseload is calculated simply as the mean value of the total bounds. The regulation capacity is calculated as absolute difference between baseload and higher (or lower) regulation bound. Capacity and baseload are finally sent to the market. This strategy allows the bounds calculator to 1) widen or reduce the regulation capacity based on batteries' SOC, 2) ensure high tracking quality, and 3) size, in a best effort manner, regulation bounds during the whole day to maximize revenues and meet all the constraints.

\subsection{Real Time Regulation Tracking Controller}

The regulation market starts to send, in a fine grain timescale, the regulation signal to the regulation tracking controller after regulation capacity and baseload are sent to the market. The tracking controller is responsible to manage CPU frequencies and batteries feeding rates. The final goal is to minimize the regulation tracking error and maximize the PHEVs' SOC.

The tracking controller 1) solves an optimization problem to minimize the tracking error, 2) calculates the amount of energy to charge PHEV batteries, and 3) calculates the total charging/discharging power for UPS to further minimize the error. The optimization finds the optimal value of aggregated CPU frequencies (the normalized mean of all the frequencies), to respect response time constrain and workload queue stability. The charging of the electric cars has higher priority over UPS to maximize PHEVs final SOC. In general, batteries in the set $R$ with lower energy have more priority over others in the same set with higher SOC. In addition, the UPS with highest energy in the set $D$ is the first to be discharged. This algorithm allows in many cases to have zero tracking error. On the other hand, unpredictable variations of workload and regulation signal may lead to unexpected quicker termination of batteries' cycles. The loss of the batteries may result in small tracking errors. However, as we show in Section 8.4, this gap is less than $6 \%$ and thus acceptable according to market rules.

\section{Power Management System}

In this section, we first describe in detail the hourly regulation bounds calculator. We then describe the real time regulation tracking controller.

\subsection{Bounds Calculator}

Constraints on control inputs and unpredictability of regulation signal heavily affect the regulation capacity. The number of active servers needs to be well decided to avoid overprovisioning or over-subscription. As Figure 3(a) shows, the bounds calculator decides the contributions of servers, PHEVs, and UPS to regulation capacity independently by each other. In particular, it determines 1) the contribution of DVFS based on average workload, response time and queue stability, and 2) the contribution of batteries proportionally to their current SOC. The regulation upper bound $R_{u p}(k)$ is calculated as

$$
R_{u p}(k)=D_{u p}(k)+R_{P, u p}(k)+R_{U, u p}(k)
$$

where $D_{u p}(k)$ is the DVFS upper bound. $R_{P, u p}(k)$ and $R_{U, u p}(k)$ denote the contribution to $R_{u p}(k)$ of PHEVs and UPS, respectively. The lower bound $R_{\text {low }}(k)$, is calculated as:

$$
R_{\text {low }}(k)=D_{\text {low }}(k)-R_{U, \text { low }}(k)
$$

where $D_{\text {low }}(k)$ is the DVFS lower bound. $R_{U, l o w}(k)$ is the contribution of UPS to the regulation lower bound. The power baseload $P_{b}$ is then calculated as the average value of $R_{u p}(k)$ and $R_{\text {low }}(k)$. The regulation capacity is the absolute difference between the baseload and $R_{u p}$ or $R_{\text {low }}$. Next three sections we describe in detail how these components are calculated. 


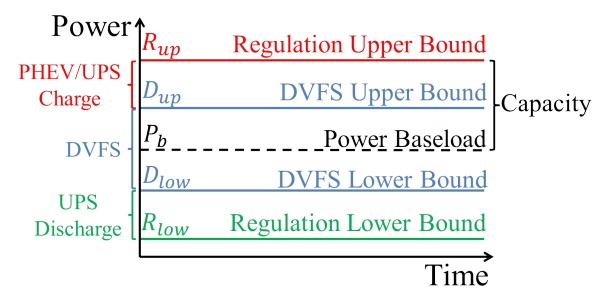

(a) Servers and batteries contribute to widening regulation bounds.

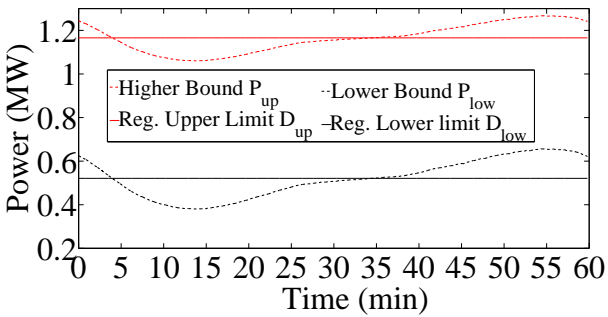

(b) DVFS regulation bounds are calculated as average of max and min server power consumption.

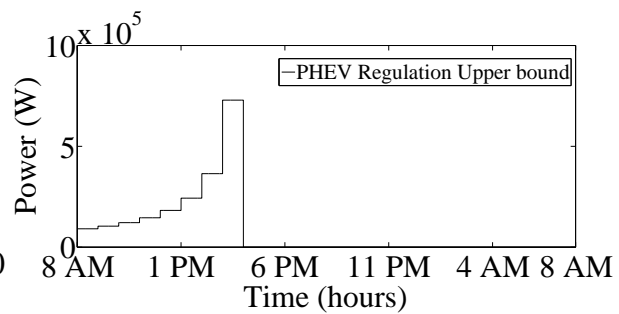

(c) PHEV regulation upper bound is calculated proportionally to batteries energy levels.

Figure 3: Bounds for regulation capacity.

\subsubsection{DVFS Bounds and Capacity Provisioning}

We decide $D_{u p}(k)$ and $D_{\text {low }}(k)$ according to the servers that on average are necessary to comply with the response time constrain. We regard the data center as a queuing system with generalized distributions on service time, request arrival time and request size. By Allen-Cullen approximation for $\mathrm{G} / \mathrm{G} / \mathrm{N}$ models [33], [34], the average response time $R_{T}(k)$ is:

$$
R_{T}(k)=\frac{1}{u(k)}+\frac{C_{A}^{2}+C_{B}^{2}}{2} \frac{\left(\rho(k)^{N_{a}(k)}+\rho(k)\right) / 2}{N_{a}(k) u(k)-\lambda(k)}
$$

where $u(k)$ is the per-server mean service rate and $N_{a}(k)$ the number of active servers. We assume that $u(k)$ is proportional to the average of normalized frequencies of all the active servers (e.g., $u(k)=k f(k)$ ). $\lambda(k)$ is the normalized workload arrival rate for the entire data center. $C_{A}$ and $C_{B}$ are the squared coefficients of variation of request inter-arrival time and request size, respectively. $\rho(k)$ is the average utilization of the servers:

$$
\rho(k)=\frac{\lambda(k)}{N_{a}(k) u(k)}
$$

To ensure stability of the queue, we enforce that:

$$
N_{a}(k) u(k)-\lambda(k)>0
$$

Note that in Equation (3) $N_{a}(k)$ has only a negligible impact on the response time of the data center. The first term $1 / u(k)$ has indeed much more weight and thus we consider that $R_{T}(k) \approx$ $1 / u(k)$. The term $1 / u(k)$ hence, is used to determine a lower aggregated frequency that respects the desired response time. Inequality (5) determines the number of active servers to respect queue stability. Define a scaling factor $\gamma$ :

$$
\gamma=\frac{k N_{a}(k)}{\lambda_{\text {mean }}(k)}
$$

$\gamma=1$ indicates that all the servers must be set at the maximum frequency level just to serve the average incoming requests. Both workload and regulation signal involve substantial uncertainties. Therefore, the bounds calculator introduces redundancy in number of active servers to process the workload peak and to follow the regulation signal. The choice of $\gamma$ is thus a trade-off between performance and energy cost. We show the influence of the scaling factor in Section 8.4. Once we have chosen a fair value for $\gamma$ (depends on the workload variations), we calculate $N_{a}(k)$ from Equation (6).
We calculate the value of $D_{u p}$ and $D_{l o w}$ based on the number of active servers and based on the following server power consumption model [19]:

$$
P_{i}(k)=\alpha_{i} f_{i}(k) \lambda_{i}(k)+\beta_{i}
$$

where $P_{i}(k)$ is the power consumption of the $i^{\text {th }}$ server. $f_{i}(k)$ is the normalized frequency and $\lambda_{i}(k)$ the normalized workload of $i^{\text {th }}$ server. In order to balance different servers power consumption, we first assign the highest frequency (at most 1) to the servers with the smallest coefficient $\alpha_{i}$; then to the second smallest $\alpha_{i}$, and so on, until the average frequency is $f(k)$ (the aggregate frequency $\left.f(k)=\sum_{i}^{N_{a}(k)} \frac{f_{i}(k)}{N_{a}(k)}\right)$. In this way we get the individual frequencies of each server, as well as the total power consumption

$$
P_{s}(k)=P\left(\lambda(k), N_{a}(k), f(k)\right) .
$$

To set the regulation limits $D_{\text {up }}$ and $D_{\text {low }}$, we define a higher bound $P_{u p}(k)$ and a lower bound $P_{l o w}(k) . P_{u p}(k)$ is the actual maximum power consumption with $f(k)=1$. $P_{\text {low }}(k)$ is the actual minimum power consumption with $f(k)$ calculated as the maximum between:

$$
\left\{\begin{aligned}
\frac{1}{u(k)} & <R s \\
f(k) & >\frac{\lambda(k)}{\gamma \cdot \lambda_{\text {mean }}}
\end{aligned}\right.
$$

where $R s$ is the maximum tolerance on response time. The first inequality ensures the respect of SLAs and the second one the queue stability. Since we assume to know only the average of the workload, $D_{u p}$ and $D_{\text {low }}$ are assigned to the average of $P_{u p}(k)$ and $P_{\text {low }}(k)$, respectively, as shown in Figure $3(\mathrm{~b})$. This choice allows to have a regulation capacity neither too conservative nor too aggressive.

\subsubsection{PHEV Bounds}

The calculation of $R_{P, u p}(k)$ is based on PHEVs' energy level and remaining working hours. The bounds calculator has to 1) maximize the final energy of PHEVs, 2) respect the limited time in which cars are on-site, and 3) minimize the tracking error.

Consider the simplification to have homogeneous arrival and departure times for all the employees between 8 AM and 4 PM. Consider also the worst regulation signal $P_{r e g}^{\text {worst }}$ equivalent to $R_{P, u p}(k)$ for half an hour (only half because the average of regulation signal $P_{r e g}$ over one hour is statistically 
Table 1: Amount of UPS energy used for regulation in every period.

\begin{tabular}{|c|c|c|}
\hline & $S O C(0)>50 \%$ & $S O C(0)<50 \%$ \\
\hline$t_{1}$ & $\mathrm{SOC}(0)$ & $100 \%-\operatorname{SOC}(0)$ \\
\hline$t_{2}-t_{1}$ & \multirow{2}{*}{$100 \%$} & $100 \%$ \\
\cline { 3 - 3 } $24-t_{2}$ & & $100 \%$ \\
\hline total capacity & $100 \%+\mathrm{SOC}(0)$ & $300 \%-\mathrm{SOC}(0)$ \\
\hline
\end{tabular}

the baseload $P_{b}$ ). Because the value of the regulation signal is unknown, the value of $R_{P, u p}(k)$ is calculated based on the assumption that $P_{\text {reg }}=P_{\text {reg }}^{\text {worst }}$. This conservative strategy is necessary to minimize tracking errors. However, this choice may lead to a low final SOC for PHEV cars because of the fluctuations of the regulation signal. Therefore, the bounds calculator first, splits the charge of PHEVs among the 8 working hours, and second, widens hour by hour the upper bound $R_{P, u p}(k)$ depending on actual PHEV energy. Figure 3(c) shows an example of $R_{P, u p}(k)$. This algorithm allows to correct the difference between $P_{\text {reg }}$ and $P_{\text {reg }}^{\text {worst }}$ to have more chances to charge the cars. On the other hand, if batteries are almost full, $R_{P, u p}(k)$ should be consequently closer to zero. Therefore, we calculate $R_{P, u p}(k)$ as

$$
R_{P, u p}(k)=\left\{\begin{array}{cc}
2 \frac{N_{p}(k) B C_{\max }^{P}-\sum_{i=1}^{N_{p}(k)} B C_{i}^{P}(k)}{8-k} & \text { if } k \in[0,7], \\
0 & \text { if } k \in[8,23] .
\end{array}\right.
$$

where $N_{p}(k)$ is the number of PHEV batteries that are currently charging. $B C_{\max }^{P}$ and $B C_{i}^{P}(k)$ are respectively the maximum and the $i^{\text {th }}$ actual battery capacity. This mechanism 1) conservatively maximizes the bound $R_{P, \text { up }}(k)$ during the working hours to maximize revenues, 2) ensures the necessary energy to track (at least in average) any fluctuation of $P_{\text {reg }}$, and 3) adapts the regulation capacity to maximize, in a best effort manner, the final SOC of cars.

\subsubsection{UPS Bounds}

The last step is to calculate the two bounds $R_{U, u p}(k)$ and $R_{U, \text { low }}(k)$. The strategy applied is similar to the one described above for PHEVs. The bounds calculator updates $R_{U, u p}(k)$ and $R_{U, l o w}(k)$ hourly, to compensate fluctuations of regulation signal based on current SOC. However, UPS can also be discharged. This characteristic stems two problems: how to preserve battery lifetime and how to schedule the charging/discharging cycles during the day to maximize revenues. Consider that PHEVs are on site for limited time and that often the DVFS is not enough to receive high revenues (as shown Section 8.5). In order to maximize profits, the data center needs to provide regulation service for 24 hours. UPS batteries have the role to widen the regulation capacity as much as possible during all day.

As suggested in [13] and [32], we do not deplete the batteries. We instead establish a maximum Depth of Discharge (DoD). Moreover, we limit the number of charges to 2 and discharges to 1 . In order to align charging/discharging cycles and profits maximization, we divide the 24 hours in charging/discharging periods. In particular, a UPS battery with initial SOC less than $50 \%$, is placed in the charging set $R$ at the beginning of the day; otherwise, the UPS is places in the set $D$. Every
UPS in the set $R$, if never discharged, is allowed to switch in $D$ when fully charged; otherwise, the UPS becomes unavailable until the next day. Similar case for switching from $D$ to $R$ with the exception that the maximum DoD has to be respected. Figure 4(a) illustrates an example of this strategy. The equations used to calculate $R_{U, \text { up }}(k)$ and $R_{U, l o w}(k)$ are similar to Equation (10). For reason of space they are not reported but Figure 4(b) shows an example. The last step the bounds calculator has to do, is to estimate conservatively the length of each cycle to redistribute all the energy during the whole day. Define $t_{1}$ as the end of the first cycle and $t_{2}$ as the end of the second one. We calculate $t_{1}$ and $t_{2}$ as the fraction of capacity that the battery can offer to the regulation during that period. Referring to the general rule in table 1 , a battery with initial SOC lower than $50 \%$ has:

$$
\begin{aligned}
& t_{1}=24 \frac{100-\operatorname{SOC}(0)}{\text { total capacity }} \\
& t_{2}=t_{1}+24 \frac{100}{\text { total capacity }} .
\end{aligned}
$$

The battery can be charged between $\left[0, t_{1}\right]$, discharged between $\left(t_{1}, t_{2}\right]$ and finally charged again until the end of the day. However, as for the PHEVs, $R_{U, \text { up }}(k)$ and $R_{U, l o w}(k)$ are calculated based on a worst regulation signal $P_{\text {reg }}^{\text {wort }}$. Because of fluctuations of regulation signal, UPS may not have finished the charging or discharging cycle at the end of each period. Hence, the bounds calculator extends hour by hour $t_{1}$ and $t_{2}$, if necessary, until the battery is full or reaches the minimum allowed (see Section 8.3).

This policy distributes the energy of the UPS through the whole day to maximize the regulation revenues and ensure tracking quality. However, unpredictability of regulation signal and workload, may generate unexpected charges and discharges of UPS. In these cases batteries may finish their cycles faster then expected, such that small tracking errors occur.

\subsection{Tracking Controller}

Once the capacity is decided and sent to the market, the latter starts to send with fixed time-step the regulation signal $P_{r e g}(k)$ to the tracking controller. The target of the real time tracking controller is to ensure that

$$
P_{g}(k)=P_{s}(k)+R(k)-D(k) \equiv P_{r e g}(k)
$$

where $P_{g}(k)$ is the total power from the grid. $P_{s}(k)$ is the total power demanded by servers. $R(k)$ and $D(k)$ are the total power charged to or discharged from batteries.

In general, the controller distributes the power as follow (Figure 3(a)):

- if $P_{\text {reg }}(k) \in\left[D_{\text {low }}, D_{\text {up }}\right]$ then $P_{s}(k)=P_{\text {reg }}(k)$

- if $P_{r e g}(k) \in\left(D_{u p}, R_{u p}\right]$ then $R(k)=P_{r e g}(k)-P_{s}(k)$ where $P_{s}(k)=D_{u p}$

- if $P_{\text {reg }}(k) \in\left[R_{\text {low }}, D_{\text {low }}\right)$ then $D(k)=P_{s}(k)-P_{\text {reg }}(k)$ where $P_{s}(k)=D_{\text {low }}$

However, if the workload presents large variation around its hourly average, there could be exceptions on this rule. Consider 


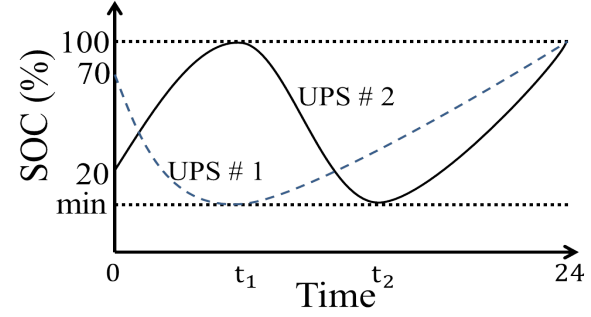

(a) The schedule of UPS charge and discharge cycles depends on initial SOC.

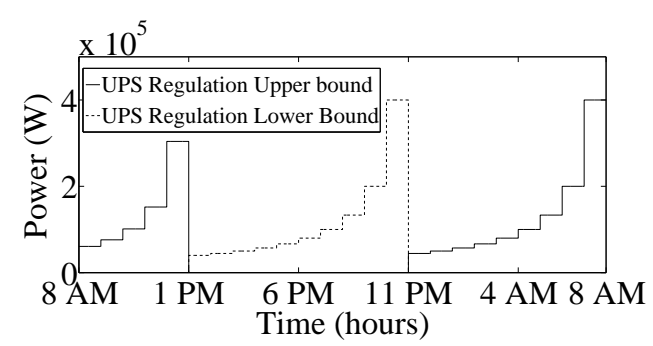

(b) UPS regulation upper and lower bounds are calculated proportionally to batteries energy levels.

Figure 4: UPS cycle schedule and bounds for regulation capacity.

for example whether, in order to comply with SLAs, $P_{s}(k)$ is higher than $P_{r e g}(k) \in\left(D_{u p}, R_{u p}\right]$. In this case, UPS have to be discharged of $D(k)=P_{s}(k)-P_{\text {reg }}(k)$ rather than charged to eliminate the tracking error. These special cases are studied in Section 8.4. The cycles of the batteries end faster than expected. Simulation results of Section 8.4 however, indicate that the tracking error never exceeds $6 \%$, which is sufficiently good since the regulation market allows small tracking errors.

\subsubsection{Tracking with DVFS}

We apply DVFS to follow the regulation signal, such that response time and queue stability are respected. Considering the aggregated power model (8), the tracking problem can be formulated as

$$
\min _{f(k)}\left|P_{r e g}(k)-P_{s}(k)\right|
$$

subject to:

$$
f(k) \in[0,1], R_{T}(k)<R s, \text { and } N_{a}(k) u(k)-\lambda(k)>0 .
$$

Regulation bounds $D_{u p}$ and $D_{\text {low }}$ are carefully chosen according to the above constraints. Hence, in most cases the regulation signal can be accurately followed by DVFS. However, occasional peaks or valleys of workload lead to compensating the gap with batteries.

\subsubsection{Tracking with DVFS and PHEV}

In all the cases in which $R(k)>0$, the tracking controller needs to distribute the total charge $R(k)$ among batteries. Consider that cars are on-site for a limited time and that we want to charge PHEVs as much as possible. The tracking controller gives priority to PHEV's batteries over UPS, to cover the entire gap $R(k)$.

A characteristic that we consider for PHEVs, is the fairness of charging, which implies two facts: 1) PHEVs with low SOC have higher priority to be charged, and 2) all the cars have to receive some charge. In order to realize a policy that complies with these two characteristics, the tracking controller calculates the total energy $\operatorname{Tot}_{c}(k)$ that remains to charge among all PHEV batteries. Then, the controller decides the charging power of every car as the minimum among:

- $R(k) \frac{B C_{\max }^{P}-B C_{i}^{P}(k)}{\operatorname{Tot}_{c}(k)}$ (fair charge)

- $R_{\max }^{P}(\max$ charge $)$
- $S T\left(B C_{\max }^{P}-B C_{i}^{P}(k)\right)$ (in case applying the previous two would exceed the battery capacity)

where $S T=\frac{3600}{4}$ is to convert the energy into power (time step of 4 seconds). $B C_{\max }^{P}$ is the maximum capacity of the battery and $B C_{i}^{P}(k)$ is the current energy level of the $i^{t h}$ battery. $R_{\max }^{P}$ is the maximum power applicable. Naming the total power used to charge PHEVs as $R_{t o t}^{P}(k)$, it is possible that $R(k)-R_{t o t}^{P}(k)$ is nonzero. In this case we fill up the gap with the UPS.

\subsubsection{Tracking with DVFS, PHEV and UPS}

The tracking controller as final step, needs to distribute the remaining charge $R(k)-R_{t o t}^{P}(k)$ or the discharge $D(k)$ among the UPS. The distribution is similar to the PHEVs. The difference is that it depends on the current time-range and energy level. Hence, starting with the $i^{\text {th }}$ UPS battery in $R$ with lowest energy (or with the $i^{t h}$ UPS battery in $D$ with highest energy), the power charged (or discharged) is equal to the minimum among:

- $R(k)-R_{\text {tot }}^{P}(k)(D(k)$ if discharge $)$

- $R_{\max }^{U}\left(D_{\max }^{U}\right.$ if discharge $)$

- $S T\left(B C_{\max }^{U}-B C_{i}^{U}(k)\right)\left(S T\left(B C_{i}^{U}(k)-B C_{\min }^{U}\right)\right.$ if discharge).

where $R_{\max }^{U}$ and $D_{\max }^{U}$ are the maximum power charged to and discharged from UPS, respectively. $B C_{\max }^{U}$ and $B C_{\min }^{U}$ are energy upper and lower bounds of UPS. This procedure is iterated in case that only one battery is not enough.

\section{Thermal Energy Storage and Cooling Power}

In this section we show how to modify our power management system to make it consider also the cooling power of the data center. The cooling system represents a big portion $(30 \%$ to $40 \%$ [35]) of the total data center power demand. In order to 1) have backup for the chillers in case of failure and 2) save on OpEx, modern data centers supplement the chiller with a Thermal Energy Storage device (TES) such as water or ice tank [36]. When the temperature of water or ice of these tanks is sufficiently low, they can substitute totally or in part the chiller in supplying chilled coolant to the Computer Room Air Conditioning unit (CRAC). This combination allows to reduce chiller's power. Therefore, the phase in which TES are used 
to save chiller power is also called discharging mode (from the point of view of the TES). On the other hand, the TES requires additional power to cool down its water or ice in the case they are not cold enough. Therefore, this phase is called charging mode of the TES. Both charging and discharging modes of TES can be exploited in the regulation market to further increase revenues for a data center. We now describe how to modify first the regulation capacity and second the tracking controller for the addition of the TES in the power management system.

A common way to calculate the cooling power demand of a data center involves the Coefficient Of Performance (COP) of the cooling system [37]. In particular the cooling power can be related with the server power as $P_{\text {cool }}(k)=P_{s}(k) / C O P$. Assuming that the TES is sized to support the entire chiller for a certain period of time (the minimum is generally 12 minutes [36]), the maximum power that the TES can discharge is given by $P_{T E S}^{\max }=P_{s}^{\max } / C O P$, where $P_{s}^{\max }$ is the maximum total power consumption of servers. Translating the thermal energy of the TES to the electric energy consumed (charge mode) or saved (discharge mode), we can treat the TES as a UPS. However TES's lifetime does not have any relation with the frequency of charge and discharge cycles. We name $E_{T E S}^{\max }$ the maximum amount of energy that the TES can charge and with $E_{T E S}^{\min }$ the minimum energy necessary to ensure reliability of the TES. For simplicity we consider that $P_{T E S}^{\max }$ is also the maximum power that the TES can draw in charging mode. At the beginning of each regulation hour $k$, we decide whether the TES should be discharged or charged based on the information on average cooling power demands in next hour. Specifically, the TES is discharged if:

$$
E_{T E S}(k)-E_{T E S}^{\min } \geq \frac{D_{u p}(k)+D_{\text {low }}(k)}{2} \frac{1}{C O P} 1 h
$$

where $E_{T E S}(k)$ is the energy level of the TES at time $k . D_{u p}(k)$ and $D_{\text {low }}(k)$ are the bounds calculated in Section 5.1.1. The left component of Inequality 15 is the energy that can be discharged using TES. The right component is instead the average energy demand of the cooling system in next hour. Therefore the TES is discharged if it can support the total average power demand and is charged otherwise. We name with $R_{T, u p}$ the additional contribute of TES to the regulation upper bound of Equation 1. Moreover, $R_{T, \text { low }}$ is the additional contribute of TES to the regulation lower bound of Equation 2. Therefore, we calculate these two bounds from the maximum or minimum energy that can be charged or discharged from TES in next hour: (considering that the regulation signal has statistical mean value equal to zero over 1 hour):

$$
\begin{aligned}
R_{T, \text { up }}(k) & =2 \frac{E_{T E S}^{\max }-E_{T E S}(k)}{1 h} \\
R_{T, \text { low }}(k) & =2 \frac{E_{T E S}(k)-E_{T E S}^{\min }}{1 h}
\end{aligned}
$$

In order to use the TES to track the regulation signal we need to define what is the priority for charging or discharging tanks respect servers, electric cars, and UPS. Note that the consideration of TES requires us to consider the cooling power be- sides server demand. Hence from now on if we use the TES in the regulation we calculate the servers power demand as the sum of computational power plus cooling power, such that the total power demand of servers is $P_{s}(k)(1+1 / C O P)$. Because 1) TES does not have limitations on daily usage as UPS batteries and 2) electric cars still have priority to charge. We assign lower priority to charge or discharge TES respect electric cars and higher priority respect UPS. The charging power $R(k)-R_{\text {tot }}^{P}(k)$ (residual from electric cars charge) assigned to TES is hence calculated as the minimum among:

$$
\begin{aligned}
& \text { - } R(k)-R_{t o t}^{P}(k) \\
& \text { - } P_{T E S}^{\max } \\
& \text { - } S T\left(E_{T E S}^{\max }-E_{T E S}(k)\right)
\end{aligned}
$$

Naming with $R_{\text {tot }}^{T}(k)$ the total power assigned to charge TES, the eventual residual capacity $R(k)-R_{\text {tot }}^{T}(k)$ is handled by UPS batteries as showed in Section 5.2.3. The discharge policy is quite similar to the charge policy and hence for reasons of space we choose to omit it.

\section{Simulation Strategy}

In this section we briefly describe workload and regulation signals used for the simulations. In addition, we define two baselines used to show the effectiveness of our strategy.

Figure 5(a) shows the normalized 24 hours Google trace [38]. The trace varies during the day between $20 \%$ and $90 \%$, with the peak reached smoothly at 10 PM. Figure 5(b) instead, shows the Wikipedia trace [39], which is characterized by high variations around the average. Because both workloads present a clear daily pattern, we assume the availability of an estimated average workload rate based on past data.

Figure 5(c) reports the first two hours of the regulation signal from PJM [40]. We do not report the entire 24 hours because of its high variability. The trace is normalized between 1 and -1. At the beginning of each operation hour hence, this signal is adapted to upper and lower regulation bounds.

In order to demonstrate the effectiveness of our solution, we use two common baselines in our experiment:

- DVFS, which tracks regulation signal only with DVFS;

- DVFS+UPS, which adds the UPS to the DVFS to widen the regulation capacity and track the regulation signal.

These baselines help to show the impact of using different knobs on the data center regulation revenues.

\section{Results}

\subsection{Experiment Setup}

To test our control system, we consider 1) one data center with 1.5MW of critical power, 2) 6,250 servers, each one $240 \mathrm{~W}$ maximum power, and 3) 3 UPS batteries each one $250 \mathrm{kWh}$ capacity and $500 \mathrm{~kW}$ maximum power. Moreover we consider to 


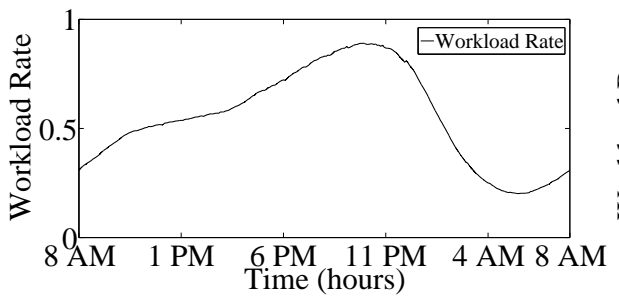

(a) Normalized Google workload trace.

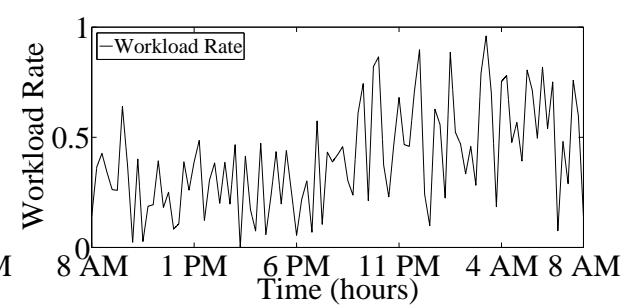

(b) Normalized Wikipedia workload trace.

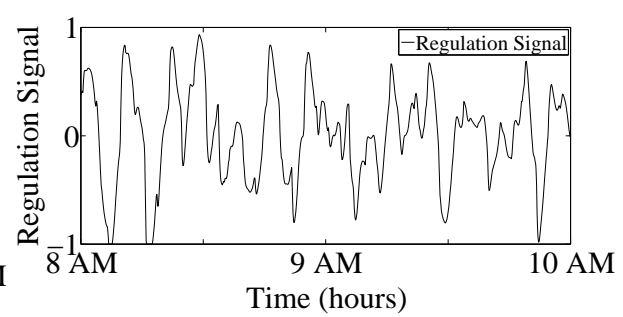

(c) First 2 hours of normalized regulation trace.

Figure 5: Real-world workload and regulation traces.

have 100 on-site PHEV vehicles with batteries of $10 \mathrm{kWh}$ capacity and $5 \mathrm{~kW}$ power. We assume that modern data centers have between 50 to 200 employees. We first use the Google trace and the regulation signal described in Section 4. Then, we present the test with the Wikipedia trace to show the impact of workload variation on the tracking error. We then show a return of investment study, in which we change data center size, number of cars and regulation price to compare revenues with different configurations. Finally we present a study to show the influence of the scaling factor $\gamma$ on performance.

In all the simulations, the constrain on the response time (6 $\mathrm{ms}$; can be chosen arbitrarily) is not violated; we avoid to report the figure for reasons of space.

\subsection{Bounds and Tracking with Google trace}

In this section we first show how SOC of batteries and workload influence regulation bounds. Then, we compare the regulation capacity of the power management system with two baselines.

Unpredictable fluctuations of the regulation signal can cause, in some period of the day, a slow charge of batteries. As Figure 6 and 7 show, between 5 PM to 10 PM regulation upper bounds of servers and batteries are different; however, the regulation signal is mostly below the DVFS bound and consequently, batteries receive a low charge. On the other hand, the bounds calculator widens, when necessary, regulation bounds to maximize final SOC of PHEVs and to distribute UPS energy through the whole day. As Figure 7 shows indeed, between 8 AM to 2 PM and $1 \mathrm{AM}$ to $8 \mathrm{AM}$, the power management system distributes more power to batteries than servers. This strategy allows a complete charge of PHEVs (see Section 8.3). The workload directly influences the power baseload, which increases in case of request peaks and decreases in case of valleys. The tracking error is zero during the whole day. The results demonstrate that our scheme 1) efficiently adapts regulation bounds to SOC of batteries and workload, and 2) provides high quality regulation service.

Figure 8 shows the difference in regulation capacity percentage between our control strategy and two commonly used baselines. At the beginning of the day, our algorithm doubles the increment in capacity given by DVFS+UPS. Our scheme obtains significant results also during the evening, when it increases capacity about $30 \%$ compared to the only DVFS baseline. The results indicate that our scheme outperforms commonly used baselines by providing higher regulation capacity and consequently, higher regulation revenues (see Section 8.5).

\subsection{Batteries cycles with the Google trace}

In this section we show how our scheme manages PHEV and UPS batteries during regulation service.

Figure 9(a) shows the energy level variation of PHEV batteries, and Figure 9(b) their final SOC. Our algorithm fairly charges batteries, such that PHEVs with lower energy receive a faster charge than the ones with higher energy. All the batteries reach the maximum capacity 2 hours before the deadline (4 PM). This fast charge is due to the presence of UPS batteries, which further widen the regulation upper bound contribution of PHEVs. Consequently, the power dedicated to charging the cars, can be higher than the estimated worst case $P_{\text {reg }}^{\text {worst }}$. Figure 9(c) shows that, between 8 AM to 1 PM, the UPS 1 does not receive any charge. This battery indeed, starts at $8 \mathrm{AM}$ in the charging set $R$ and should switch to the discharging set $D$ around $2 \mathrm{PM}\left(t_{1}=6\right.$ hours). However, at $2 \mathrm{PM}$ the energy in the UPS ${ }_{1}$ still needs to be used (because of the priority to charge given to PHEVs). Therefore, the bounds calculator extends the permanence of this battery in the set $R$. This strategy allows to manage UPS to last for the whole day in order to maximize regulation revenues. Moreover, UPS energy never exceeds the maximum depth of discharge ( $80 \%$ for this simulation). The results demonstrate that our algorithm 1) provides a complete charge of PHEV batteries before the deadline, 2) leverages daily UPS cycles to maximize revenues, and 3) preserves lifetime of batteries.

\subsection{Evaluation with the Wikipedia Trace}

Figure 10 shows results of the simulation made with the Wikipedia trace. As explained in Sections 4 and 5, in general our algorithm decides to charge or discharge batteries depending on the power zones (Figure 3(a)). However the Wikipedia trace presents high variability around its average value (which is used to calculate DVFS bounds). This characteristic leads to the possibility to have unexpected discharges in the charge zone, or unexpected charges in the discharge zone. The tracking error in Figure 10(c), is zero until there is at least one battery that can be discharged and one that can be charged. After 10 PM for example (Figures 10(a), 10(b)), it is impossible to have zero tracking error because all the batteries are charging. Indeed, there is nothing to discharge when the server power consumption is above the regulation signal. However, Figure 10(c) shows that this error (averaged every 5 minutes) is below $6 \%$. This result is sufficiently good because the regulation market allows small tracking errors. This simulation test hence, indicates that our 


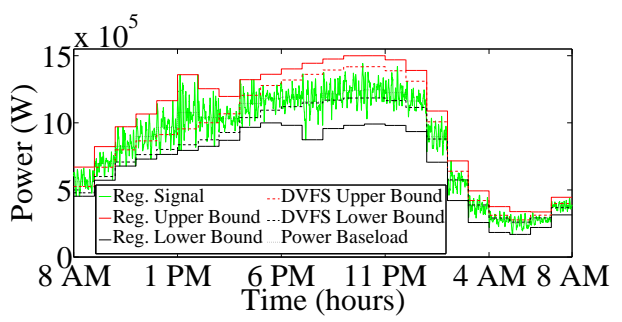

Figure 6: The regulation bounds are carefully calculated to comply with batteries' energy and workload.

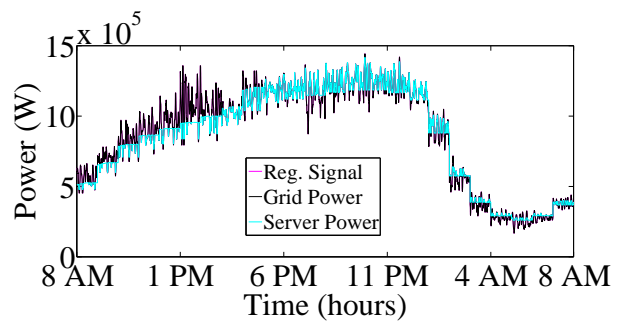

Figure 7: The algorithm distributes grid power among servers and batteries to track the regulation signal.

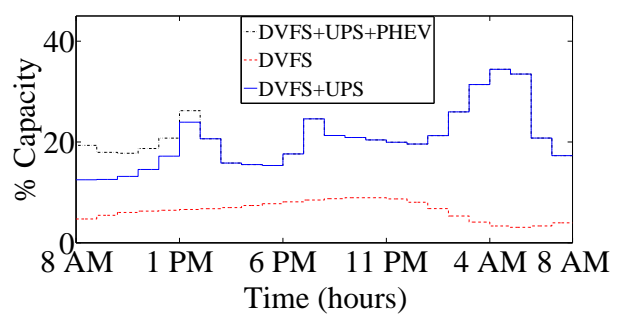

Figure 8: The proposed solution (DVFS+UPS+PHEV) leads to more regulation capacity than two baselines.

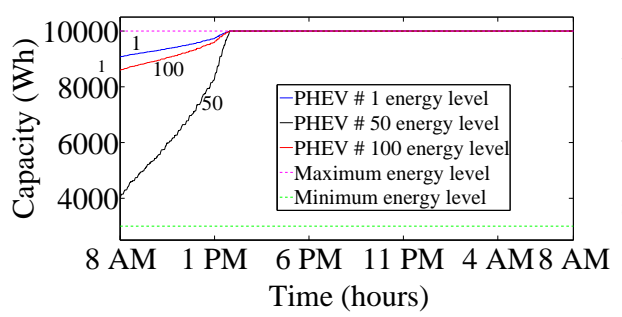

(a) The charging rate of every PHEV depends on the current energy level.

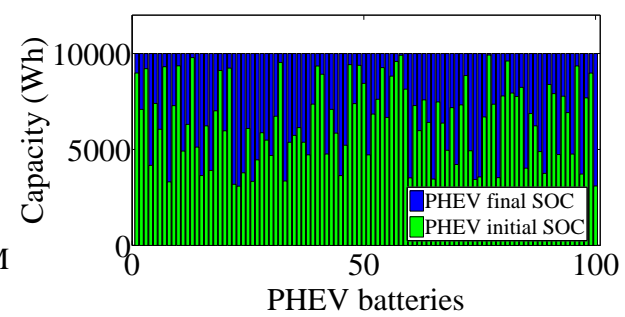

(b) The proposed solution leads to a complete charge of PHEV batteries.

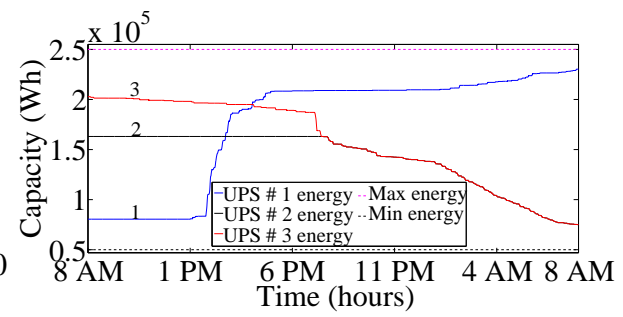

(c) The charging and discharging rates of every UPS depend on the current energy level.

Figure 9: Energy of PHEV and UPS batteries in the regulation service with the Google workload.

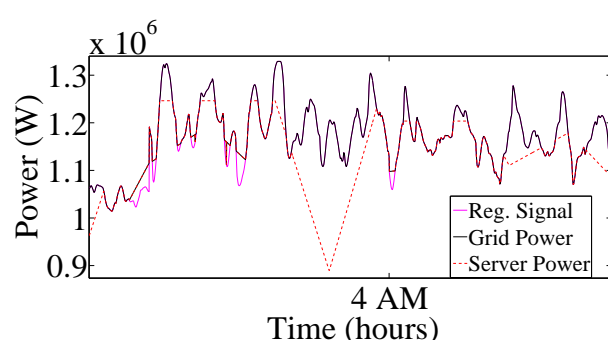

(a) Fluctuations of workload lead to quick termination (b) In order to compensate workload fluctuations, of batteries' cycles and to small tracking errors.

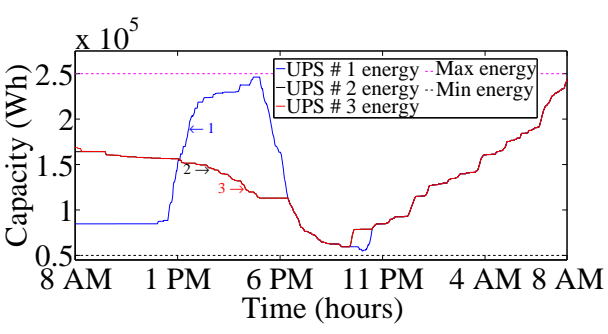

UPS batteries rapidly finish their cycles.

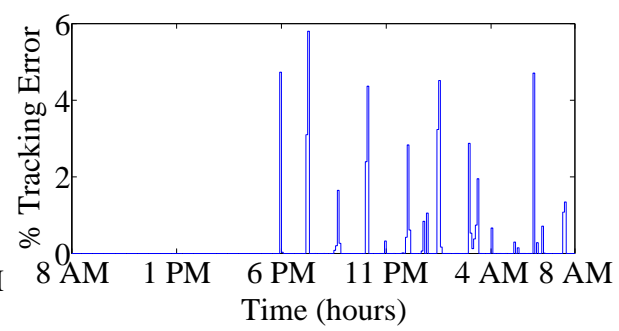

(c) Small tracking errors occur because of workload fluctuations.

Figure 10: Simulation test for the Wikipedia trace.

Table 2: Return of investment over 15 years for PHEV owners. Electricity price is $\$ 50 / \mathrm{MWh}$ from [41], fuel expense from [42] and PHEVs' DoD 70\%

\begin{tabular}{|c|c|c|c|c|}
\hline & Car & $\begin{array}{c}\text { Fuel } \\
(1 \text { year })\end{array}$ & $\begin{array}{c}\text { Energy } \\
(1 \mathrm{y} .)\end{array}$ & $\begin{array}{c}\text { Gains } \\
(15 \mathrm{y} .)\end{array}$ \\
\hline GAS & 0 & $\$ 1,100$ & 0 & $/$ \\
\hline PHEV & $\$ 6,000$ & 0 & $\$ 128$ & $\$ 8,580$ \\
\hline PHEV+data center & $\$ 6,000$ & 0 & 0 & $\$ 10,500$ \\
\hline
\end{tabular}

algorithm keeps high tracking quality even in presence of high fluctuations in the incoming workload.

\subsection{Return of Investment}

Here we first study how the regulation revenues vary for different configurations: 1) different data centers' critical power $(0.5,1,2,3$ and $5 \mathrm{MW})$ with regulation price fixed at \$50/MWh [40], 2) different knobs used, 3) total number of daily regulation hours (8 and 24 hours), 4) different number of on-site PHEVs, and 5) different regulation prices. Then, we analyze the cost savings of the PHEV owners, throughout the lifetime of the PHEVs, by getting their batteries charged at no expense.

Figure 11(a), shows regulation revenues of our solution compared to two baselines (DVFS and DVFS+UPS), for different data centers. In average PHEVs' contribution allows revenues to experience an annual increase of $15 \%$ (8 regulation-hour per day) over the baseline DVFS+UPS. These gains decrease for the 24 hours case. Indeed, while UPS batteries allow an increase on revenues of $71 \%$ over the baseline DVFS, the gains for the PHEV batteries are not remarkable (about 5\%). This result is due to the fact that an increase of critical power does not necessarily correspond to an increase on the number of cars or employees (we consider a maximum of 200 employees). However, considering the case of a small data center (0.1MW, 50 PHEVs; the results are not reported for reasons of space), our scheme leads to an increase in revenues between $30 \%$ to $50 \%$ over the DVFS+UPS baseline. In this case, the total energy of PHEVs is comparable with the data center critical power. In order to understand the impact of the PHEVs on revenues, we report in Figure 11(b) the percentile increment of regulation revenues for different numbers of on-site PHEVs. Revenues increase from $9 \%$ to $28 \%$ in case of 8 hours regulation per day, and from $3 \%$ to $10 \%$ in the other case. Another factor that affects regulation profits, is the regulation price. Figure 11(c) shows how revenues increase when regulation price varies between $\$ 30$ and \$50/MWh [40]. In all the cases presented, our control system outperforms the baselines by having higher reg- 

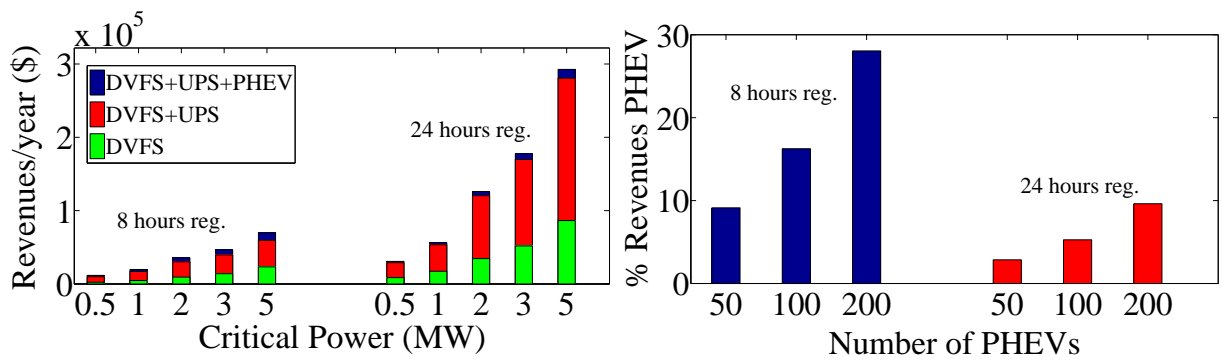

(a) The proposed solution (DVFS+UPS+PHEV) leads (b) Increasing the number of on-site PHEVs leads to to more regulation revenues than two baselines, for a higher weight of the cars on regulation revenues. all data centers of different sizes.

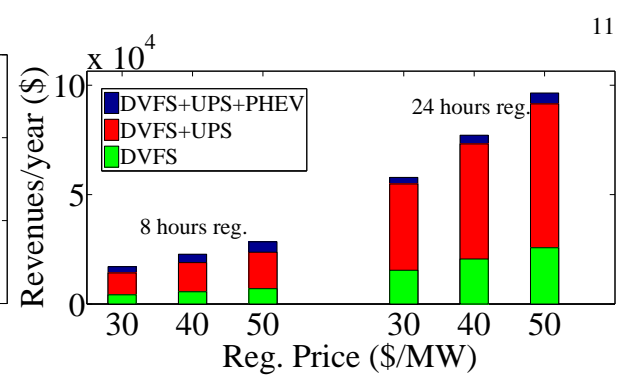

(c) The proposed solution (DVFS+UPS+PHEV) leads to more regulation revenues than two baselines, for all the regulation prices.

Figure 11: Regulation revenues for data centers under different configurations and different per-day regulation hours.

Table 3: Aggregated frequency ranges and average over 24 hours for different values of $\gamma$.

\begin{tabular}{|c|c|c|c|}
\hline$\gamma$ & $f_{\min }$ & $f_{\max }$ & average \\
\hline 1.2 & 0.85 & 1 & 0.96 \\
\hline 1.7 & 0.6 & 1 & 0.84 \\
\hline 2 & 0.54 & 1 & 0.82 \\
\hline
\end{tabular}

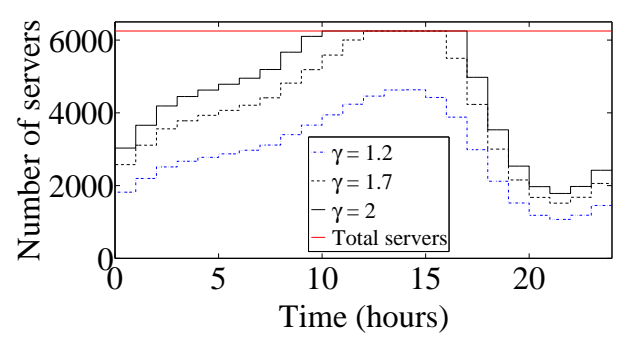

Figure 12: The choice of the scaling factor $\gamma$ is a trade-off between robustness of the data center to workload fluctuations and energy consumption.

ulation revenues. The results indicate that our scheme, allows a $1.5 \mathrm{MW}$ data center with 100 on-site PHEVs, to receive about $\$ 95,000$ from regulation service (20\% of annual electricity bill). Moreover, profits considerably increase with data center size, number of on-site PHEVs and regulation price.

Table 2, shows the Return Of Investment (ROI) for PHEV owners. Compared to normal gas-cars, they gain about $\$ 8,580$ over the car lifetime. However, our solution increases savings of data center's employees to $\$ 10,500$ per car. Assume that there are 100 on-site electric cars. The combination data center and PHEVs allows an entire fleet of vehicles to increase savings of about $\$ 192,000$, by getting batteries charged at no expense.

\subsection{Influence of the scaling factor}

In this section we describe how the scaling factor $\gamma$ (Equation (6)), affects performance of our system. In particular, $\gamma$ influences number of active servers and CPU frequencies.

Figure 12 and Table 3 show how number of active servers and normalized aggregated CPU frequency $f(k)$ change, when $\gamma$ assumes values in the set $\{1.2,1.7,2\}$. When the scaling factor is low, the workload is processed with a low number of servers. Although this choice leads to decreasing data center energy consumption, the aggregated frequency is in average equivalent to 0.96 . Therefore, this configuration allows only small variations of workload from its average value without performance degradation. When $\gamma$ is high instead, the number of active server is higher than the previous case, as well as the energy consumption of the data center. However, $f(k)$ decrease

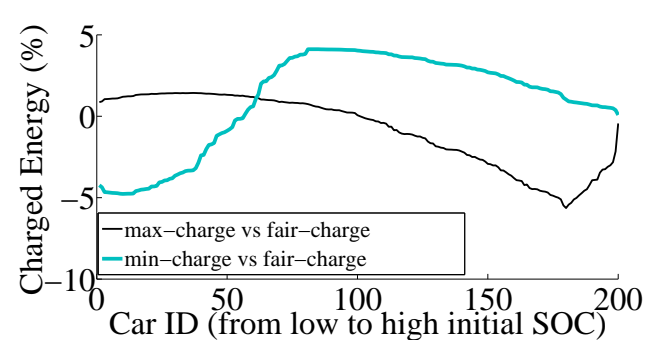

(a) Only PHEV

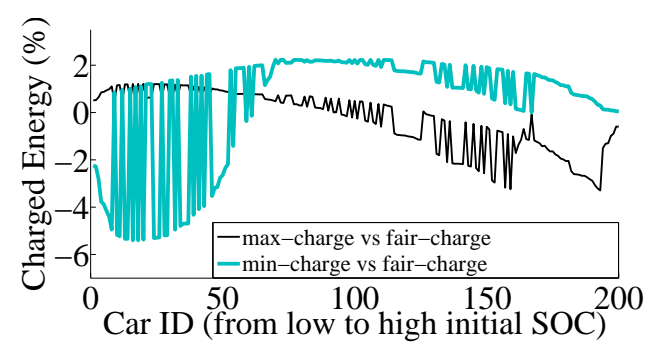

(b) Half PHEV and half BEV

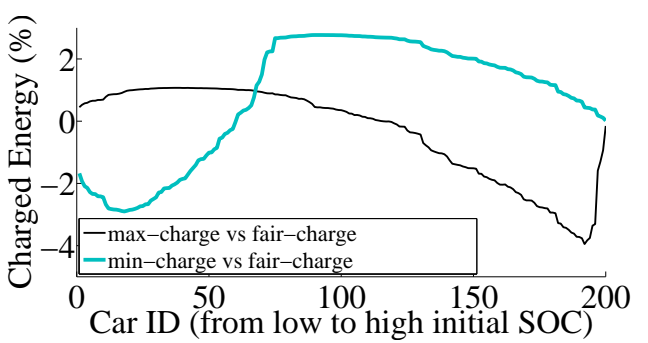

(c) only BEV

Figure 13: Percentage of charged energy during the 8 working hours. No matter what kind of electric vehicle is on-site, our fair policy ensures the distribution of power to all the cars and a faster charge for those with lower SOC.

to 0.82 and hence, higher variations of the workload around its average value are allowed. The results indicate that the scaling factor is a trade-off between robustness of the data center to workload variations and energy consumption. We plan in our future work to formulate an optimization problem that finds an optimal value for $\gamma$.

\subsection{Different Types of Electric Vehicles}

As said at the end of Section 3.2, Battery Electric Vehicles (BEV) can also be used in our scheme. Different by PHEVs, BEVs are only equipped with an electric engine. In order to maintain a certain drive range, these cars are equipped with a 


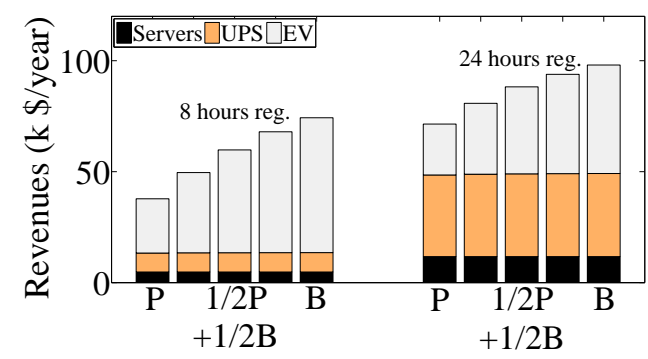

(a) Regulation revenues for different mixtures of EVs. From $\mathrm{P}=100 \% \mathrm{PHEV}$ to $\mathrm{B}=100 \% \mathrm{BEV}$

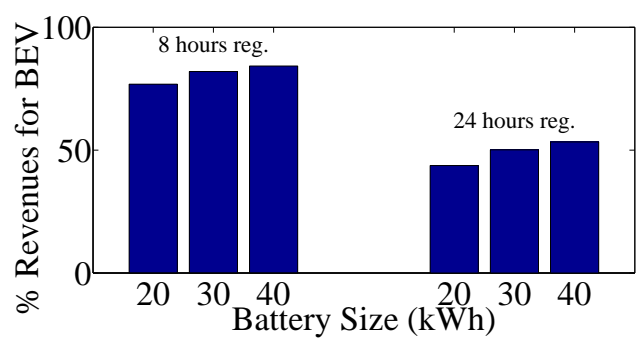

(b) Cars' battery size weight on revenues.

Figure 14: Different types of EV and battery size influence the weight of electric vehicles over the total revenues.

bigger battery (usually between $20 \mathrm{kWh}$ to $40 \mathrm{kWh}$ against the $10 \mathrm{kWh}$ of the PHEVs). In this section we consider a mixture of 200 electric cars with different portions. Moreover we define two baselines, max-charge and min-charge. The first one charges the batteries with lower SOC as fast as possible, while the second one charges equally all the cars. We have defined these two baselines because they represent two opposite extremes compared with our fair policy (as we explain later). First, we compare the benefits of our fair-charge policy with the two baselines. Second, we show how PHEVs and BEVs impact the regulation revenues for the data center.

Figure 13 shows a comparison in terms of energy charged to EVs at the end of the 8 working hours. In the figure, EVs have been sorted from low to high initial SOC and the two lines show the percentage of the mathematical difference of energy charged to cars with max-charge or min-charge and energy charged with our fair-charge. The cars for which this difference is positive, receive more energy with the corresponding baseline than with our fair policy. In particular, Figures 13(a), 13(b), and 13(c) show three simulation results considering 200 PHEVs, 100 PHEVs plus $100 \mathrm{BEVs}$, and $200 \mathrm{BEVs}$, respectively. Maxcharge dedicates as much power as possible to charge the cars with lower SOC (cars in the left side of the figures) which lead to a higher amount of energy charged respect our fair-policy. However, max-charge never distribute power to some of the cars with higher initial energy (cars on the right side of the figures), while our policy ensures to distribute some energy to all the cars. Min-charge redistributes equally the power among all the batteries but does not consider the actual state of charge of each car. This policy therefore, inevitably leads to a high difference in final SOC among different cars. Indeed the cars with low initial SOC (left side of figures) can not charge their bat- teries fast enough to reach an acceptable SOC before the end of the day, while the EVs with high initial SOC (right side of figures) get more chances to complete their cycle on time. Our fair policy balances at same time the benefits of max-charge and min-charge. This fact is showed in the figures by observing that the two curves cross each other at a certain point. Our fair policy performs better than max-charge but worse than min-charge in terms of energy charged to cars with high SOC. However our policy performs better than min-charge but worse than maxcharge in terms of energy charged to cars with low SOC. This it means that our fair policy reduces the total charge dedicated to EVs with high SOC to allow a faster charge of EVs with low SOC, yet ensuring the distribution of some power to all the cars. These results do not change with the type of electric cars present on the data center site or with a mixture of PHEVs and BEVs. In the case of the mixture however, the big difference in battery size leads the PHEVs to complete their charge cycle before the BEVs (fluctuations in Figure 13(b)).

Figure 14(a) shows the total regulation revenues for different portions of PHEVs (10kWh batteries) and BEVs (30kWh) present on-site. Figure 14(b) shows the weight of BEVs on regulation revenues for different battery sizes. As one can notice from these two figures, compared to PHEVs the bigger size of BEV's batteries leads to a higher increase of the regulation revenues. In particular as the portion of BEVs increases respect PHEVs, regulation revenues increase from $\$ 37,800 /$ year to $\$ 74,300 /$ year considering only the 8 regulation hours in which cars are on-site, and from $\$ 71,500 /$ year to $\$ 98,100 /$ year in the case of 24 regulation hours. Therefore 8 hours of regulation with 200 on-site BEVs can lead even to more revenues than 24 hours regulation with 200 on-site PHEVs. Moreover, BEVs become even more important than UPS also in the case of 24 hours of regulation since now they account for the bigger portion of regulation revenues. Figure 14(b) shows that, as expected, also the size of these cars influences total revenues. Batteries' size can lead to increasing the percentage of regulation revenues due to the usage of cars from $77 \%$ to $85 \%$ for 8 hours of regulation and from $44 \%$ to $54 \%$ for 24 hours of regulation. These results indicate that electric cars heavily impact the regulation revenues of a data center. Therefore, a data center participating in the regulation market may increase revenues by reserving part of them to incentive its employees on buying an electric car.

\subsection{Thermal Energy Storage in Regulation Market}

In this section we show how the power management system works when the thermal energy storage is used as additional knob for regulation. Here we consider the presence of 100 onsite $30 \mathrm{kWh} \mathrm{BEVs}$, and a thermal tank sized to sustain the entire cooling system to supply temperature at 20 degree Celsius for maximum 30 minutes. Moreover, we set 2 UPS and the TES with initial energy such that they start the day in the discharging set, and 1 UPS starting in the charging set. First we discuss the influence of TES on batteries energy levels, and second we discuss their influence on regulation revenues. 


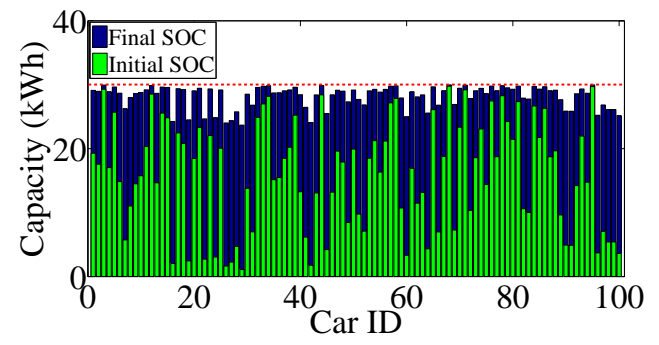

(a) Final energy level of 30kWh BEV's batteries.

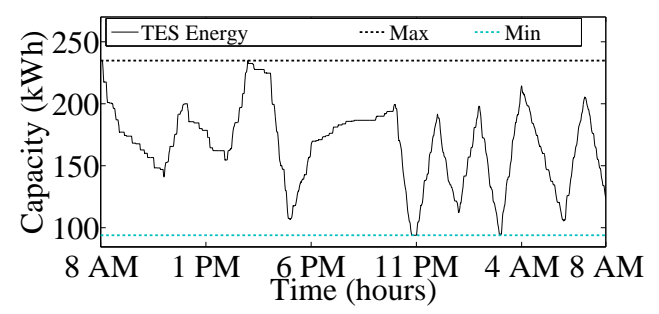

(b) Variation of energy charged and discharged using TES

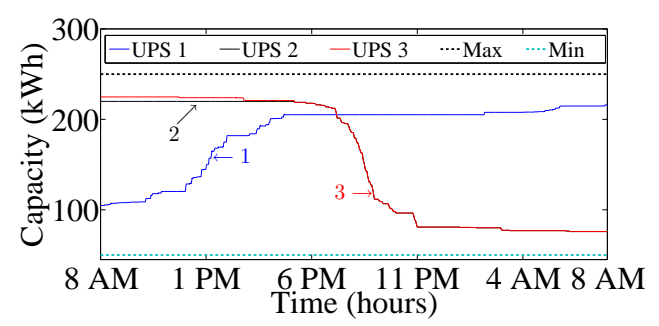

(c) Variation of energy level of UPS batteries

Figure 15: Our solution combines electric cars, TES, and UPS to achieve high regulation tracking quality while ensuring high final energy level for large-sized BEV's batteries, reliability of TES, and lifetime of UPS.

Figure 15 shows the energy variation of the knobs considered (BEV, TES, and UPS). In particular Figure 15(a) shows initial and final state of charge of BEVs. Even though the fluctuations of the regulation signal and the big size of the batteries do not allow a complete charge for all the cars, our solution still leads to a high average final energy level. Figure 15(b) indeed shows that the TES is charged much slower during the first 8 hours in which cars are on site respect the hours in which they leave. This variation of charge (and discharge) rate allows first to give more priority for the charge of electric cars, and second alleviates the necessity of UPS, of which energy variation is showed in Figure 15(c). It is worth to remind that the reduction of the usage of UPS batteries has two advantages: first, lifetime is further protected and second, their energy can be used for longer periods of time to reduce tracking errors. Indeed if the UPS are less used they stay longer in the initial charge or discharge set. This fact leads to almost zero tracking errors for both workloads Google and Wikipedia (see discussion in Section 8.4).

Figure 16 shows the variation of regulation upper and lower bounds of servers, BEV, TES, and UPS. Figure 17 shows how the TES influences regulation revenues. As described in previous sections, when the servers contribute and the upper or lower bounds of regulation capacity overlap each other, the probabil-

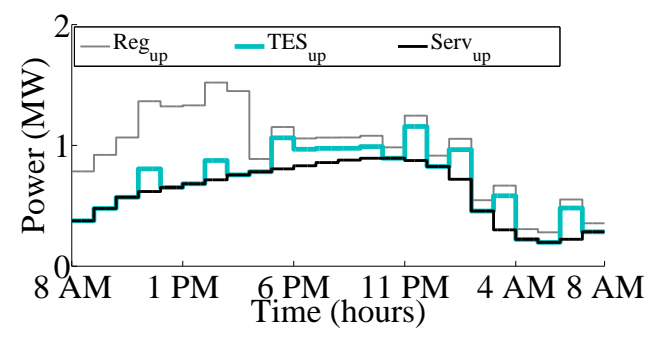

(a) Regulation Upper Bound

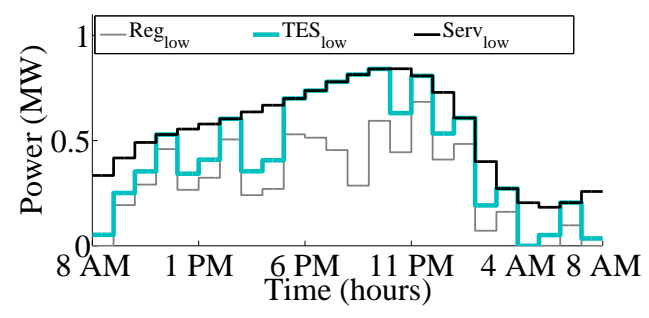

(b) Regulation Lower Bound

Figure 16: Regulation Bounds. TES, UPS, and Cars help to increase the total regulation capacity.

ity of tracking error increases. The presence of the TES and the longer presence of UPS in their initial set allow to have for most of the time an additional upper and lower bound around the capacity given by servers. Moreover, as Figures 17(a) and 17(b) show, the TES can heavily increase both the percentage of regulation capacity (up to $80 \%$ more than only server and UPS) and regulation revenues (up to $\$ 87,000$ more revenues respect only server and UPS).

\section{Conclusions}

Frequency regulation is an ancillary service designed to reduce the undesired imbalance between power supply and demand in the energy market. In order to participate in the regulation market, both the supply and demand sides need to be capable of flexibly adjusting their power generation and consumption, respectively. Internet data centers, due to their increasing power consumption, are able to participate in the regulation market to minimize operating expenses through regulation profits. On the other side, PHEVs are also eligible participants in the regulation market, due to their large power demand for battery charging. In this paper, we proposed a novel power management scheme that jointly leverages a data center and its employees' PHEVs to 1) maximize the revenues that the data center receives from the regulation market, and 2) get the PHEVs charged at no expense to their owners. Our simulation results based on different real traces, demonstrate that our scheme leads to high quality of regulation, and to a complete charge for PHEVs. As a result, the proposed scheme outperforms commonly used baselines by having higher regulation revenues for the data center. A return of investment study, shows that with our algorithm, 1) a $1.5 \mathrm{MW}$ data center can lower OpEx by gaining about $\$ 95,000 /$ year of regulation revenues (20\% of annual electricity bill), and 2) each PHEV owner save about $\$ 10,500$ over the car lifetime. A fleet of 100 cars therefore, experiences an increase of $\$ 192,000$ for the free 


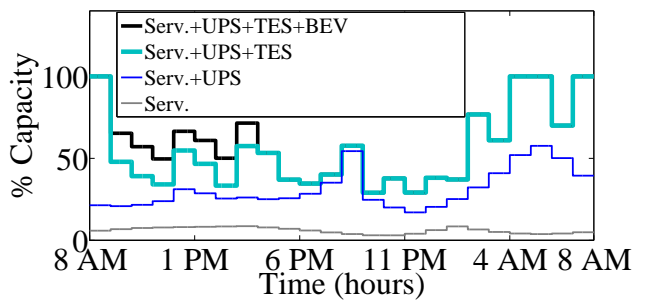

(a) Comparison of percentage regulation capacity for different solutions

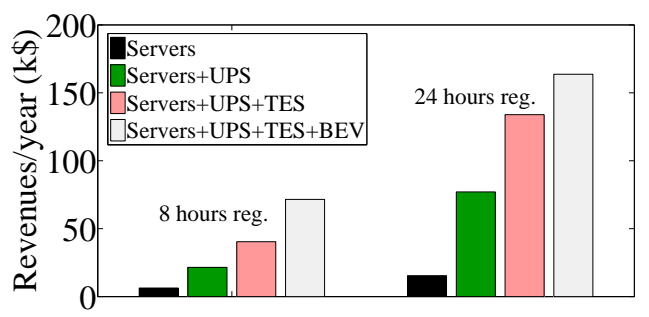

(b) Comparison of regulation revenues for different solutions

Figure 17: The addition of TES further increases regulation capacity and revenues.

charge from the data center. Moreover, the integration of TES in the regulation problem and the consideration of electric cars with bigger batteries allow to increase total revenues to about $\$ 165,000 /$ year while minimizing average tracking error. These increased gains may be reinvested by the data center to stimulate its employees on buying electric cars. In this way cars' owners can experience a higher return of electric vehicle investment, while the data center can exploit the higher number of on-site EVs to further boost regulation revenues.

\section{References}

[1] E. Hirst and B. Kirby, "Electric-power ancillary services," Consult kirby, 1996. [Online]. Available: http://consultkirby.com

[2] A. Keech, "PJM manual 12: Balancing operations," PJM, 2012.

[3] N. Y. I. S. Operator, "Ancillary services manual," NYISO, 2012.

[4] M. C. D. of PJM, "PJM manual 28: Operating agreement accounting," PJM, 2012.

[5] C. I. S. Operator, "Business requirements specification-pay for performance regulation," CAISO, 2012.

[6] N. Y. I. S. Operator, "Accounting and billing manual," NYISO, 2013.

[7] R. Wang, N. Kandasamy, and C. Nwankpa, "Data centers as demand response resources in the electricity market: Some preliminary results," Feedback Computing, 2012.

[8] D. Aikema, R. Simmonds, and H. Zareipour, "Data centers in the ancillary services market," IGCC, 2012.

[9] W. Kempton, V. Udo, K. Huber, K. Komara, and S. Letendre, "A test of Vehicle-to-Grid (V2G) for energy storage and frequency regulation in the PJM system," V2G, 2008.

[10] F. K. Tuffner and M. Kintner-Meyer, "Using electric vehicles to mitigate imbalance requirements associated with an increased penetration of wind generation," PES, 2011.

[11] A. Ulbig, M. D. Galus, S. Chatzivasileiadis, and G. Andersson, "General frequency control with aggregated control reserve capacity from timevarying sources: The case of PHEVs," IREP, 2010.

[12] X. Wang, M. Chen, C. Lefurgy, and T. W. Keller, "Ship: Scalable hierarchical power control for large-scale data centers," PACT, 2009.

[13] V. Kontorinis, L. E. Zhang, B. Aksanli, J. Sampson, H. Homayoun, E. Pettis, D. M. Tullsen, and T. S. Rosing, "Managing distributed ups for effective power capping in data centers," 39th International Symposium on Computer Architecture, 2012.
[14] Z. Liu, M. Lin, A. Wierman, S. H. Low, and L. L. H. Andrew, "Greening geographical load balancing," SIGMETRICS', 2011.

[15] EPA, "Report to congress on server and data center energy efficiency, public law 109-431," U.S. Environmental Protection Agency ENERGY STAR Program, 2007.

[16] P. X. Gao, A. R. Curtis, B. Wong, and S. Keshav, "Its not easy being green," SIGCOMM, 2012.

[17] J. Weinman, "Why data centers have a big impact on the economy," gigaom, 2012. [Online]. Available: http://gigaom.com/

[18] R. Miller, "How many servers can one admin manage," Datacenter Knowledge, 2009. [Online]. Available: http://www.datacenterknowledge. com

[19] C. Lefurgy, X. Wang, and M. Ware, "Server-level power control," ICAC, 2007.

[20] X. Wang and M. Chen, "Cluster-level feedback power control for performance optimization," HPCA, 2008.

[21] F. Ahmad and T. N. Vijaykumar, "Joint optimization of idle and cooling power in data centers while maintaining response time," ASPLOS', 2010.

[22] R. Urgaonkar, B. Urgaonkar, M. J. Neely, and A. Sivasubramanian, "Optimal power cost management using stored energy in data centers," SIGMETRICS, 2011.

[23] H. Hao, T. Middelkoop, P. Barooah, and S. Meyn, "How demand response from commercial buildings will provide the regulation needs of the grid," CSL, 2012.

[24] A. Molina-Garcia, F. Bouffard, and D. S. Kirschen, "Decentralized demand-side contribution to primary frequency control," IEEE Transactions on Power Systems, 2011.

[25] C. Li, C. Ahn, H. Peng, and J. Sun, "Integration of plug-in electric vehicle charging and wind energy scheduling on electricity grid," ISGT, 2012.

[26] B. Kirby, "Ancillary services: Technical and commercial insights," Report prepared for Wartsila, 2007.

[27] hybridCARS, "A comprehensive guide to plug-in hybrids," Hybridcars, 2009. [Online]. Available: http://www.hybridcars.com

[28] K. Smith, M. Earleywine, E. Wood, J. Neubauer, and A. Pesaran, "Comparison of plug-in hybrid electric vehicle battery life across geographies and drive cycles," SAE, 2012.

[29] K. Smith, A. J. Markel, and A. A. Pesaran, "PHEV battery trade-off study and standby thermal control," 26th International Battery Seminar \& Exhibit, 2009.

[30] T. Lee, Z. Bareket, T. Gordon, and Z. S. Filipi, "Stochastic modeling for studies of real-world PHEV usage: Driving schedule and daily temporal distributions," IEEE TRANSACTIONS ON VEHICULAR TECHNOL$O G Y, 2012$

[31] H. Lee and G. Lovellette, "Will electric cars transform the U.S. vehicle market? an analysis of the key determinants," Cambridge, Mass.: Belfer Center for Science and International Affairs, Tech. Rep., 2011-08.

[32] S. Govindan, A. Sivasubramaniam, and B. Urgaonkar, "Benefits and limitations of tapping into stored energy for datacenters," ISCA, 2011.

[33] A. Allen, "Probability, statistics, and queueing theory with computer science applications," Academic Pr, 1990.

[34] G. Bolch, S. Greiner, H. de Meer, , and K. Trivedi, "Queueing networks and markov chains: modeling and performance evaluation with computer science applications," Wiley-Interscience, 2006.

[35] Y. Zhang, Y. Wang, , and X. Wang, "TEStore: Exploiting thermal and energy storage to cut the electricity bill for datacenter cooling," CNSM, 2012.

[36] D.Garday and J. Housley, "Thermal storage system provides emergency data center cooling," Intel Information Technology White Paper, 2007.

[37] J. Moore, J. Chase, P. Ranganathan, and R. Sharma, "Making scheduling cool: Temperature-aware workload placement in data centers," USENIX, 2005 .

[38] Google, "Google transparency report." [Online]. Available: http: //www.google.com

[39] W. Foundation, Wikimedia, 2007. [Online]. Available: http://download. wikimedia.org/

[40] PJM, "Pjm regulation data." [Online]. Available: http://www.pjm.com

[41] “2013 pjm electricity historical prices,” Futures Trading Charts. [Online]. Available: http://futures.tradingcharts.com

[42] Cars101, "Fuel economy yearly costs." [Online]. Available: http: //www.cars101.com 